\title{
Effects of valence and arousal on written word recognition: Time course and ERP correlates
}

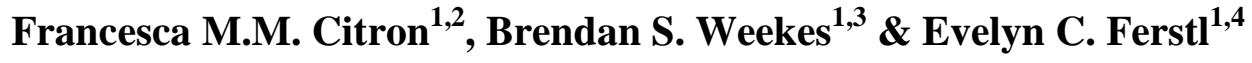

${ }^{1}$ School of Psychology, University of Sussex, Pevensey Building, Falmer, BN1 9QH, UK

${ }^{2}$ Cluster Languages of Emotion, Freie Universität Berlin, Habelschwerdter Allee 45, 14195

Berlin, Germany

${ }^{3}$ Laboratory for Communication Science, The University of Hong Kong, Pakfulam Road, Hong Kong

${ }^{4}$ Center for Cognitive Science, Albert-Ludwigs-Universität Freiburg, Friedrichstrasse 50, 79098 Freiburg, Germany

Address correspondence to:

Francesca Citron

Cluster Languages of Emotion

Freie Universität Berlin

Habelschwerdter Allee 45

D-14195 Berlin

Tel.: $\quad+49(0) 3083857869$

Fax: $\quad+49(0) 3083852887$

E-mail:fmm.citron@gmail.com

Word count: 4,473

Short title: Valence and arousal effects on ERPs 


\begin{abstract}
Models of affect assume a two-dimensional framework, composed of emotional valence and arousal. Although neuroimaging evidence supports a neuro-functional distinction of their effects during single word processing, electrophysiological studies have not yet compared the effects of arousal within the same category of valence (positive and negative). Here we investigate effects of arousal and valence on written lexical decision. Amplitude differences between emotion and neutral words were seen in the early posterior negativity (EPN), the late positive complex and in a sustained slow positivity. In addition, trends toward interactive effects of valence and arousal were observed in the EPN, showing larger amplitude for positive, high-arousal and negative, low-arousal words. The results provide initial evidence for interactions between arousal and valence during processing of positive words and highlight the importance of both variables in studies of emotional stimulus processing.
\end{abstract}

Key words: valence, arousal, ERP, EPN, LPC, slow positivity, lexical decision, word processing. 


\section{Introduction}

Theoretical accounts of emotional stimulus processing assume a two-dimensional structure of affect: valence describes whether an emotional stimulus is positive or negative, whereas arousal refers to intensity of emotion $[17,23]$. Recent literature reviews $[3,14]$ show that studies of written word processing report faster and more accurate recognition of words with emotional valence compared to neutral words; in addition, emotion words elicit a larger amplitude in event-related potential (ERP) components associated with implicit emotion processing $[13,16,24,27]$. Interestingly, the effects are observed on tasks that do not require emotional judgement including written lexical decision (LD; deciding if a letter string is a word), silent reading and letter detection. Since these tasks do not require selective attention to a stimulus' emotional content, the effects observed reflect implicit emotion processing. Instead, on tasks such as valence decision, selective attention to the emotional content of the word is involved.

The early posterior negativity (EPN, 200-300 ms) has occipito-temporal scalp distribution and shows larger amplitude for valent stimuli (positive and negative) than neutral stimuli [8, 9]; it indexes automatic, effortless attention orientation toward a stimulus emotional content [26], henceforth labelled emotionality, and it is taskindependent [14].

The late positive complex (LPC; 500-800 ms) has centro-parietal distribution and is sensitive to a stimulus valence, typically showing larger amplitude for valent than neutral stimuli $[13,26]$ or a difference in amplitude between positive and negative words [9]. It reflects resource allocation toward and enhanced processing of a stimulus emotional content [e.g., 9]; it is associated with explicit, task-related evaluation of the stimulus [5]. 
The LPC is task-dependent: emotion effects are observed for lexical decision, silent reading and semantic tasks that require processing depth, but not on less demanding tasks, e.g., judgment of font consistency [24]. LD requires less processing depth than semantic tasks, but the latter ones may direct attention towards other stimulus aspects (e.g., its imageability). Some studies reported long-lasting, centro-parietal effects, extending the LPC into a sustained slow positivity (SSP; observed from 700 to 1000 ms during word processing [6]), which reflects sustained attention to visual emotional stimuli as well as memory processes [25].

\subsection{Modulation of valence by arousal}

Interactive effects of valence and arousal are reported in analyses of LD latencies [18]. Specifically, arousal modulates speed of identification of negative words: negative low-arousal (NL) words take longer to recognise than negative highly arousing (NH) and positive words. Studies manipulating valence and arousal in valence decision tasks [22] also report interactive effects whereby positive, low-arousal (PL) and NH stimuli are responded to faster than positive, high-arousal $(\mathrm{PH})$ and NL stimuli. Such interactions support a multi-dimensional model of affect. Specifically, PL and NH categories elicit a straightforward approach vs. withdrawal reaction, whereas PH and NL stimuli both elicit conflicting approach-withdrawal reactions and are therefore more difficult to process [22].

It is not clear from the behavioural results whether valence and arousal interact at an early, implicit processing stage or at later stages of word recognition. ERP components may provide a window to answering this question. In a first study [12], participants were presented with NH, NL, PL and neutral, low-arousal words. LD was faster for NH than NL words and faster for PL than neutral words; furthermore, amplitude of an occipito-temporal negative ERP component (80-120 ms) was larger in both conditions. The authors argued 
that arousal has a different effect on recognition of positive and negative words at early stages of processing. In a second study [2], NH, NL and neutral words were embedded in sentences and presented during a semantic judgement task. Larger LPC amplitude for NH than neutral words was observed, but no difference between $\mathrm{NH}$ and NL words. The authors argued that the LPC is more sensitive to valence, given that it is assumed to reflect elaborative processes [3]. Although these early studies are revealing, they did not compare different levels of arousal within words with positive valence.

\subsection{The present study}

The aim of this study is to determine when arousal and arousal interact during word recognition. Lexical decision was used because it requires depth of processing but does not elicit selective attention to the emotional content of a stimulus. This task therefore allows investigation of the ERP components that are associated with implicit emotion processing.

Based on previous studies [9, 12, 18], we predicted faster LD latencies and larger EPN, LPC and possibly SSP components for emotion compared to neutral words; we also predicted interactive effects of valence and arousal on LD latencies and on ERP components. As this research is exploratory, we had no predictions about the components elicited.

\section{Method}

\subsection{Participants}

Thirty-one English native speakers from the University of Sussex (16 women; 19-36 years, $M=24, S D=5$ ), all right-handed, with normal or corrected-to-normal vision and without any learning disability, performed a LD task. They gave their written consent and received either course credits or $£ 5$. 


\subsection{Materials}

One-hundred and fifty words were selected from word norms [4] containing ratings for valence, arousal, familiarity, age of acquisition (AoA) and imageability. Valence ranged from -3 (very negative) to +3 (very positive); arousal, familiarity and imageability were scaled from 1 (not at all) to 7 (very high); for AoA, age ranges in years were given: 02, 2-4, 4-6, 6-9, 9-12, 12-16, older than 16. Length in letters, phonemes, syllables and frequency of use (spoken and written) were taken from the web-based CELEX [19]; neighbourhood size (N-size) and frequency (N-frequency) values were taken from the English Lexicon Project [1].

Emotionality was investigated by selecting 50 positive $(M=1.74, S D=0.36)$, 50 negative $(M=-1.51, S D=0.34)$ and 50 neutral words $(M=0.23, S D=0.42)$, matched for all word lengths, N-size, N-frequency, frequency (Log10), AoA and imageability, with all $F s(2,147)<1.66$. Neutral words were lower in arousal than valent words, whereas arousal level was matched between positive and negative words $t(98)=-.98, p=.33$.

Within valent words, half of the items were high and half low in arousal. Words in each condition were matched for all features described above $F s(3,96)<1.57$, except imageability $F(3,96)=18.39, p<.001$, which was significantly higher in high-arousal than low-arousal words. Therefore, imageability was controlled in the analyses by regressing raw reaction times (RTs) and accuracy values on imageability ratings; the standardised residuals were used as dependent variables.

The items were sampled from all grammatical categories (nouns, adjectives, verbs). This was not ideal but necessary as there is no precise differentiation between word class in English, unlike morphologically richer languages. Grammatical categories were distributed evenly across emotion conditions, but differed slightly for neutral words (mostly nouns); 
these items were either emotion-denoting words (e.g., happy, sad) or other emotionally valent words (e.g., fortune, conflict), also balanced across the conditions, whereas neutral items did not denote emotions.

One-hundred and fifty non-words were selected from the ARC Nonword Database [21] and matched to words for number of letters $t(289.22)=1.51$, ns and phonemes $t(298)=0.55$, ns.

\subsection{Procedure}

The experiment was programmed with E-Prime software. Participants were seated in an electrically shielded room in front of a CRT screen at a distance of $70 \mathrm{~cm}$. The stimuli were presented at the centre in non-capitalized, white letters on a black background; two letters subtended $1^{\circ}$ of visual angle. EEG was continuously recorded during the task in two runs, using a Geodesic sensor net (GSN) with 128 electrodes (EGI, Eugene, Oregon). Netstation software was used for data acquisition. Eye-movements and blink artefacts were monitored using two bipolar ocular electrodes. Impedance was kept below $50 \mathrm{k} \Omega$. Sampling rate was $250 \mathrm{~Hz}$. EEG was referenced online to the vertex electrode and band-passed filtered between 0.01 and $100 \mathrm{~Hz}$.

Participants were asked to wash and brush their hair before the application of a GSN to their head. They were instructed not to move during the recording, to avoid horizontal eye-movements and to blink only after each trial, when an eye-blink prompt was presented on the screen. They were required to read letter strings and decide whether they were English words or not, as accurately and as quickly as possible. A response box with two buttons corresponding to “yes/no” was provided and their configuration counterbalanced across participants. At the beginning of each trial, a fixation cross appeared in the centre for $800 \mathrm{~ms}$. Then a letter string appeared and remained on the screen until the LD. No time 
limit for the response was given. The screen was then blank for 1000 ms and a picture of two closed eyes appeared subsequently for $700 \mathrm{~ms}$.

A practice block of 10 trials was followed by 6 experimental blocks divided into 2 runs with a break in between. 150 words and non-words were mixed together and divided among the six blocks. Positive, negative and neutral words were equally distributed within each block. Block order and word order within blocks were randomised across participants. Accuracy, RTs and EEG responses to each item were recorded. Preparation and experiment lasted 1 hour overall.

\subsection{Data Analysis}

For each participant, outlier correction (+/-3 SDs) on RTs was applied. Only trials with correct responses were analysed ( $M 98 \%$ of the trials, SD 5\%). EEG was band-pass filtered between 0.3 and $40 \mathrm{~Hz}$ (off-line) and segmented from $100 \mathrm{~ms}$ before to $1000 \mathrm{~ms}$ after stimulus onset. Segments were baseline-corrected and re-referenced to the linked mastoids, in line with previous studies [e.g., 13]. Segments with artefacts exceeding +/- 75 $\mu \mathrm{V}$ were automatically rejected; further manual rejection was then applied, leading to overall elimination of $8 \%$ of the trials per participant.

As in previous studies [6, 9], inspection of the mean amplitude of ERPs was used to detect differences in amplitudes across conditions observed over posterior electrodes and time windows for statistical analyses were then chosen: EPN from 250 to $310 \mathrm{ms,} \mathrm{LPC}$ from 430 to 650 ms, SSP from 650 to 1000 ms. Based on their typical scalp distribution, two regions of interest (ROIs) were chosen (see Figure 2b): posterior lateral sites for EPN (electrodes 60, 67, 59, 66, 72, 65, 71 over the left hemisphere; 78, 86, 77, 85, 92, 84, 91 over the right hemisphere); midline electrodes for LPC and SSP $(62,68,73,76)$. 
Repeated-measures ANOVAs were carried out with one factor EMOTIONALITY (neutral, positive, negative); planned comparisons between valent and neutral and between positive and negative words were carried out. Additional contrasts were carried out for accuracy: positive vs. neutral and negative vs. neutral words. Within valent words, factors were VALENCE (2) x AROUSAL (2). A baseline analysis excluded effects observed due to noise in the data. Greenhouse-Geisser correction was applied in case of any violation of sphericity. Given evidence that emotion words elicit weaker but qualitatively comparable effects on brain activity than pictures $[3,10]$, marginally significant effects $(p<.1)$ were also reported, but interpreted with caution.

\section{Results}

\subsection{Behavioural Results}

3.1.1 Emotionality. Mean RT to positive words was $585.43 \mathrm{~ms}(S E=19.51)$, to negative words $587.80 \mathrm{~ms}(S E=19.87)$ and to neutral words $602.98 \mathrm{~ms}(S E=23.15)$. A main effect of emotionality $F(2,60)=5.83, p=.005, \eta^{2}=.16$ showed faster RTs for valent

than neutral words $F(1,30)=9.79, p=.004, \eta^{2}=.25$, with no difference between positive and negative words $F(1,30)=0.22$, ns.

Mean accuracy rate for positive words was 99\% ( $S E=0.3)$, for negative words $97 \%$ $(S E=0.5)$ and for neutral words $98 \%(S E=0.5)$. A main effect of emotionality $F(2,60)=$ 5.82, $p=.005, \eta^{2}=.16$ showed significantly better recognition of positive words than negative $F(1,30)=10.47, p=.003, \eta^{2}=.26$ and neutral words $F(1,30)=8.06, p=.008, \eta^{2}=$ .21. Overall, accuracy was very high and showed very low variability probably because the task is easy. Therefore, tiny differences in accuracy were statistically significant. 
3.1.2 Valence by arousal. Descriptive statistics of the standardised residuals, corrected for imageability, are shown in Figure $1(\mathrm{a}, \mathrm{b})$. A trend toward an interaction between valence and arousal in the residuals of the RTs $F(1,30)=2.97, p=.095, \eta^{2}=.09$ showed larger absolute mean residual values for PH and NL conditions than PL and $\mathrm{NH}$ conditions, indexing slower LD latencies. No main effects were found $F s(1,30)<1.52$, ns. Accuracy residuals showed a main effect of arousal $F(1,30)=7.47, p=.010, \eta^{2}=.20$, with a larger absolute mean residual value for high-arousal words, indicating greater accuracy. No other significant effects were observed $F s(1,30)<1.46$ ns.

Non-corrected RTs and accuracy rates for PH words were 577.13 ms $(S E=18.96)$ and $99 \%(S E=0.3)$, for PL words $594.21 \mathrm{~ms}(S E=20.43)$ and $98 \%(S E=0.5)$, for $\mathrm{NH}$ words $572.87 \mathrm{~ms}(S E=19.41)$ and $97 \%(S E=0.7)$, for NL words $602.53 \mathrm{~ms}(S E=20.62)$ and $97 \%(S E=0.7)$.

-INSERT FIGURE 1 ABOUT HERE

\subsection{ERP Results}

Analysis of the baseline time window revealed no significant differences among conditions for either design in both ROIs (all Fs $<1$ ). Figure 2 (a, c) shows ERPs and topographic maps for each emotionality condition. Figure 1 (c, d, e) shows the mean amplitudes of the ERP components for valent words. 
3.2.1 EPN. A main effect of emotionality $F(2,60)=3.70, p=.031, \eta^{2}=.11$ showed significantly more negative amplitude for valent than neutral words $F(1,30)=5.40, p=$ $.027, \eta^{2}=.15$ and no difference between positive and negative words $F(1,30)=.23$, ns. A trend toward an interaction of valence by arousal $F(1,30)=2.93, p=.097, \eta^{2}=.09$ showed more negative amplitude for PH and NL words than PL and NH words. No significant main effects were found $F s(1,30)<0.27$, ns.

3.2.2 LPC. A main effect of emotionality $F(2,60)=4.51, p=.015, \eta^{2}=.14$ showed more positive amplitude for neutral than valent words $F(1,30)=4.96, p=.034, \eta^{2}=.15$ and a trend toward a significantly more positive amplitude for negative than positive words $F(1,30)=3.53, p=.071, \eta^{2}=.11$. No effects of valence, arousal or their interaction were significant (all Fs $(1,30)<2.83, n s)$.

3.2.3 SSP. A main effect of emotionality $F(2,60)=9.31, p=.0001, \eta^{2}=.24$ showed more positive amplitude for neutral than valent words $F(1,30)=11.87, p=.002, \eta^{2}=.28$ and no difference within valent words $F(1,30)=1.29$, ns. A marginally significant interaction of valence by arousal $F(1,30)=3.18, p=.085, \eta^{2}=.10$ showed more positive amplitude for PL and NH words than PH and NL words. No other effects Fs $(1,30)<1.16$, ns were significant.

\section{Discussion}

The results lend support to our hypothesis that valence and arousal would have an effect on lexical decision latencies and also ERP components. We observed a prioritisation of emotionally valent words reflected by faster LD latencies and larger amplitude of the EPN, replicating previous findings [cf. 3]. We contend that emotion processing is enhanced at early stages of word recognition: EPN modulation reflects relatively automatic allocation 
of resources toward emotional stimuli and this is reflected in faster responses. Our interpretation of these effects is compatible with a two-dimensional structure of affect in current theories.

LPC and SSP components showed larger amplitude for neutral than valent words. These findings are difficult to reconcile with previous studies [14, 20]. The LPC and SSP components are task-dependent: modulation by word emotionality is not observed in some studies that employ silent reading [15] and has not been observed during less demanding tasks $[11,24,27]$. These components are more often observed in response to emotional pictures (e.g., mutilation, sexual scenes) that are very salient and more effective in engaging sustained attention [14]. These late positivities are also modulated by internal stimulus probability in oddball tasks [7] and motivational relevance or evaluative distance from a stimulus context [25]. None of these conditions for elicitation are observed in LD tasks. However, neutral stimuli might require more processing resources for performance in the present study because they are less salient and appear less frequently during the task than valent stimuli (1/3 of the words are neutral) [see also 10]. Kanske and Kotz [13] observe a classical LPC effect during LD when stimulus probability was matched between valent and neutral words. Therefore, we reason the present findings are due to a lower probability of neutral words.

Because LD requires a motor response that was performed concurrently with the appearance of late positivities, we cannot exclude the possibility that differences in amplitude may be associated with motor preparation and execution. Silent reading excludes this potential confound. We are not able to partial out the possible effects of motor control by regressing ERP amplitudes on RTs as this would require averaging single trial EEG across participants, a procedure that hinders attainment of clean ERPs and statistical power. 
We also predicted an interaction between valence and arousal on LD latencies and ERP components. Manipulation of valence and arousal found a consistent trend toward an interaction in LD latencies as reported in previous behavioural studies and more critically, this was reflected across EPN and SSP amplitudes. These results suggest that valence and arousal interact at an early, perhaps implicit stage of word recognition, due to greater allocation of attention towards PH and NL words. This is consistent with other research [12] and suggests for the first time that ERP modulation of valence by arousal can be observed for positive as well as for negative words. Furthermore, arousal modulates positive and negative valences differently. We observed a marginal interaction at later stages of processing indexed by SSP and LD latencies, engaging evaluative processes. Behaviourally, the marginal interaction seems to be driven by $\mathrm{PH}$ words, which are more slowly and accurately processed than all other conditions. However at the neural level, both the PH and NL conditions seem to be processed differently than the PL and NH conditions.

Marginal interactions on EPN and RTs replicate the results of Robinson et al. [22]. Those authors argue that stimuli which activate “conflicting” approach-withdrawal orientations (PH and NL words) require more resources and time for response than stimuli activating either approach or withdrawal (PL and NH words, respectively). We propose that conflicting orientations need to be implicitly integrated before action is programmed.. This interpretation requires confirmation from fully significant effects rather than trends.

One limitation concerns a possible confound of word imageability. It has been shown that this variable correlates with arousal [4]; therefore, imageability could not be matched between high and low-arousal stimuli. Imageability affects late, long-lasting negative components such as N400 and N700 [28], and not earlier components, where the 
marginal interaction was seen (EPN). Therefore, possible confounds between imageability and arousal on ERPs limit our interpretation of the late positivities only [13].

The inclusion of different grammatical categories might have introduced noise into the data. In fact, the onset of emotion effects on ERPs is delayed for verbs compared to nouns and adjectives [3], even though the same pattern of effects is observed across categories. Future research should compare effects of emotional variables vs. contextual stimulus frequency on late positivities during word processing.

\subsection{Conclusions}

Our findings replicate previous reports on the effects of emotional variables during word recognition. However, the present study goes beyond previous reports by showing for the first time that arousal modulates the effect of valence on ERPs during early processing of positive words. Although further research is needed to confirm our findings using better

stimuli control and more trials per condition, results support a two dimensional structure of affect. 


\section{Acknowledgments}

The University of Sussex funded this research but had no role in the conduct of the study. All authors contributed to designing the study; FMMC collected and analysed the data, interpreted the results and wrote the manuscript; BSW and ECF contributed to results interpretation and writing up. Portions of this study were orally presented at the AMLaP and Neurobiology of Language conferences (2010) and at the SPR conference (2011). 
Valence and arousal effects on ERPs

\section{References}

[1] D.A. Balota, M.J. Yap, M.J. Cortese, K.A. Hutchinson, B. Kessler, B. Loftis, J.H. Neely, D.L. Nelson, G.B. Simpson, R. Treiman, The English lexicon project, Behavior Research Methods 39 (2007) 445-459.

[2] M. Bayer, W. Sommer, A. Schacht, Reading emotional words within sentences: The impact of arousal and valence on event-related potentials, International Journal of Psychophysiology 78 (2010) 299-307.

[3] F.M.M. Citron, Neural correlates of written emotion word processing: A review of recent electrophysiological and hemodynamic neuroimaging studies, Brain and Language 122 (2012) 211-226.

[4] F.M.M. Citron, B.S. Weekes, E.C. Ferstl, How are affective word ratings related to lexico-semantic properties? Evidence from the Sussex Affective Word List (SAWL), Applied Psycholinguistics (in press).

[5] J. Dien, K.M. Spencer, E. Donchin, Parsing the late positive complex: Mental chronometry and the ERP components that inhabit the neighborhood of the P300, Psychophysiology 41 (2004) 665-678.

[6] D.G. Dillon, J.J. Cooper, T. Grent-'t-Jong, M.G. Woldorff, K.S. LaBar, Dissociation of event-related potentials indexing arousal and semantic cohesion during emotional word encoding, Brain and Cognition 62 (2006) 43-57.

[7] E. Donchin, M.G. Coles, Is the P300 component a manifestation of context updating?, Behavioral and Brain Sciences 11 (1988) 357-427.

[8] I.H.A. Franken, L. Gootjes, J.W. van Strien, Automatic processing of emotional words during an emotional Stroop task, NeuroReport 20 (2009) 776-781.

[9] C. Herbert, M. Junghofer, J. Kissler, Event related potentials to emotional adjectives during reading, Psychophysiology 45 (2008) 487-498.

[10] J.A. Hinojosa, L. Carretié, M.A. Valcarcel, C. Méndez-Bértolo, M.A. Pozo, Electrophysiological differences in the processing of affective information in words and pictures, Cognitive, Affective \& Behavioural Neuroscience 9 (2009) 173-189.

[11] J.A. Hinojosa, C. Méndez-Bértolo, M.A. Pozo, Looking at emotional words is not the same as reading emotional words: Behavioural and neural correlates, Psychophysiology 47 (2010) 748-757.

[12] M.J. Hofmann, L. Kuchinke, S. Tamm, M.L.-H. Võ, A.M. Jacobs, Affective processing within 1/10th of a second: High arousal is necessary for early facilitative processing of negative but not positive words, Cognitive, Affective \& Behavioural Neuroscience 9 (2009) 389-397. 
[13] P. Kanske, S.A. Kotz, Concreteness in emotional words: ERP evidence from a hemifield study, Brain Research 1148 (2007) 138-148.

[14] J. Kissler, R. Assadollahi, C. Herbert, Emotional and semantic networks in visual word processing: insights from ERP studies, Progress in Brain Research 156 (2006) 147-183.

[15] J. Kissler, C. Herbert, I. Winkler, M. Junghofer, Emotion and attention in visual word processing - An ERP study, Biological Psychology 80 (2009) 75-83.

[16] S.-T. Kousta, D.P. Vinson, G. Vigliocco, Emotion words, regardless of polarity, have a processing advantage over neutral words, Cognition 112 (2009) 473-481.

[17] P.J. Lang, M.M. Bradley, B.N. Cuthbert, Motivated attention: Affect, activation, and action. In: P.J. Lang, R.F. Simons, M.T. Balaban (Eds.), Attention and orienting: Sensory and motivational processes, Erlbaum, Hillsdale, NJ, 1997, pp. 97-135.

[18] R.J. Larsen, K.A. Mercer, D.A. Balota, M.J. Strube, Not all negative words slow down lexical decision and naming speed: Importance of word arousal, Emotion 8 (2008) 445-452.

[19] Max Planck Institute for Psycholinguistics, Web-based CELEX. Vol. 2007, Nijmegen, 2001.

[20] J.K. Olofsson, S. Nordin, H. Sequeira, J. Polich, Affective picture processing: An integrative review of ERP findings, Biological Psychology 77 (2008) 247-265.

[21] K. Rastle, J. Harrington, M. Coltheart, 358,534 nonwords: The ARC nonword database., Quarterly Journal of Experimental Psychology 55A (2002) 1339-1362.

[22] M.D. Robinson, J. Storbeck, B.P. Meier, B.S. Kirkeby, Watch out! That could be dangerous: Valence-arousal interactions in evaluative processing, Personality and Social Psychology Bulletin 30 (2004) 1472-1484.

[23] J.A. Russell, A circumplex model of affect, Journal of Personality and Social Psychology 39 (1980) 1161-1178.

[24] A. Schacht, W. Sommer, Time course and task dependence of emotion effects in word processing, Cognitive, Affective \& Behavioural Neuroscience 9 (2009b) 2843.

[25] H.T. Schupp, B.N. Cuthbert, M.M. Bradley, J.T. Cacioppo, T.A. Ito, P.J. Lang, Affective picture processing: The late positive potential is modulated by motivational relevance, Psychophysiology 37 (2000) 357-261. 
[26] H.T. Schupp, M. Junghöfer, A.I. Weike, A.O. Hamm, The selective processing of briefly presented affective pictures: An ERP analysis, Psychophysiology 41 (2004) 441-449.

[27] G.G. Scott, P.J. O'Donnell, H. Leuthold, S.C. Sereno, Early emotion word processing: Evidence from event-related potentials, Biological Psychology 80 (2009) 95-104.

[28] W.C. West, P.J. Holcomb, Imaginal, semantic, and surface-level processing of concrete and abstract words: An electrophysiological investigation, Journal of Cognitive Neuroscience 12 (2000) 1024-1037. 
Arousal and valence effects on ERPs

\section{Figure captions}

Figure 1. Valence by arousal design: (a) mean residuals of the imageability-corrected RTs and (b) accuracy rates; (c, d, e) mean absolute amplitude of each ERP component in form of bar graph: EPN (250-310 ms) over posterior lateral sites; LPC (430-650 ms) and SSP (650-1000 ms) over midline electrodes. For the EPN, shorter bars index larger amplitude. Error bars represent standard errors of the means.

Figure 2: (a) Emotionality design: grand-average ERPs elicited by positive, negative and neutral words, merged across electrodes in the two ROIs; (b) topographic map of the 128 electrodes: the two lateral circles index the region of interest (ROI) chosen for the EPN analysis, whereas the median circle indexes the ROI chosen for the LPC and SSP analysis; (c) topographic maps of the difference between valent words and neutral words for each relevant ERP component. 